\title{
Die erweiterte Substantivphrase in ausgewählten deutschen Wirt- schaftstexten - eine intra- und interlinguale rezeptionsgrammati- sche Analyse; Zusammenfassung einer Dissertation
}

Die betreffende Arbeit (Originaltitel: Den udvidede substantivfrase i udvalgte tyske фkonomiske fagtekster - en receptionsgrammatisk analyse i intra- og intersprogligt perspektiv $=$ Ditlevsen 1996) wurde im Sommer 1996 an der Wirtschaftsuniversität Aarhus als Dissertation angenommen (mündliche Prüfung: 4. November 1996). Die folgende Zusammenfassung ist eine überarbeitete Version von Kapitel 7 der Dissertation (Deutsches Resümee).

Die Idee zu meinem Forschungsprojekt ist im DaF-Unterricht, an dem dänischsprachige Studenten teilnehmen, entstanden, wo es immer wieder vorkom$\mathrm{mt}$, daß es den Studenten oft schwerfällt, selbst wenig komplizierte deutschsprachige Wirtschaftsfachtexte zu lesen und somit auch zu verstehen. Das Hauptziel der Dissertation ist es vor diesem Hintergrund, eine Antwort darauf zu erhalten, inwieweit morpho-syntaktische Strukturen der Substantivphrase folgende Aussagen unterstützen:

1) Wirtschaftsfachtexte aus einer Kommunikationssituation werden generell als schwerer zugänglich empfunden als Wirtschaftsfachtexte aus einer anderen Kommunikationssituation.

2) Deutschsprachige Wirtschaftsfachtexte aus einer Kommunikationssituation werden von Dänischsprachigen mit Deutsch als Fremdsprache generell als schwerer zugänglich empfunden als entsprechende dänischsprachige Wirtschaftsfachtexte.

Die Beantwortung dieser Fragen setzt eine morpho-syntaktische Beschreibung der deutschen und der dänischen Substantivphrasen aus Fachtexten der Wirtschaft voraus, welche als Ausgangspunkt für intra- und interlinguale Vergleiche dient. Verglichen werden dabei aus intralingualer Perspektive die Substantivphrasen in Fachtexten der vertikalen Schichten C und D (s. unten) und aus interlingualer Perspektive die Substantivphrasen in vergleichbaren deutsch- und dänischsprachigen Fachtexten der vertikalen Schichten C und D (s. unten). Dabei liegt das Hauptgewicht auf den deutschen Substantivphrasen. Die Analysen der dänischen Substantivphrasen dienen ausschließlich zum Vergleich, damit die aus dänischer Perspektive typischen Merkmale der deutschen Substantivphrasen deutlich zum Vorschein kommen können.

Unter Texten der vertikalen Schicht $\mathrm{C}$ verstehe ich in Anlehnung an Hoffmann 1987 Texte einer Kommunikationssituation, an der vor allem Experten (Theorie), Experten (Praxis) sowie Experten (in spe) teilnehmen; konkret han- 
delt es sich hier um Texte aus Lehrbüchern innerhalb der Betriebswirtschaftslehre. Unter Texten der vertikalen Schicht D verstehe ich wieder in Anlehnung an Hoffmann 1987 Texte einer Kommunikationssituation, an der vor allem spezialisierte Laien (i.e. Journalisten) und Experten (Praxis) teilnehmen; konkret handelt es sich hier um Texte aus Wirtschaftsmagazinen genereller Art (Wirtschaftswoche und Børsens Nyhedsmagasin).

Der Arbeit liegen zwei Annahmen zugrunde. Erstens nehme ich mit Fabricius-Hansen / Solfjeld 1994 an, daß eine unterschiedliche Verwendung von morpho-syntaktischen Strukturen in der Fremdsprache im Vergleich zur Muttersprache dazu beitragen kann, daß den DaF-Studenten das Lesen von deutschsprachigen Texten schwerer fällt. Und zweitens nehme ich an, daß die Häufigkeit und die Menge der nicht vertrauten Strukturen in einem Text mit den Schwierigkeiten korrelieren, die die DaF-Studenten beim Lese- und somit beim Verstehensprozeß haben.

Den theoretischen Rahmen der Arbeit bildet die traditionelle Grammatik, nur wird sie durch eine rezeptive Sicht ergänzt, wie sie von Heringer 1989 und Ahlgren / Fabricius-Hansen 1986 vertreten wird. Die rezeptive Sicht ist eine logische Folge davon, daß die Verständlichkeit von Fachtexten der Wirtschaft das Kernobjekt der zu beantwortenden Fragen ist.

Innerhalb dieses theoretischen Rahmens wird das Analysemodell, das rezeptionsgrammatische Modell, vorgestellt, wobei es auf der Basis der deutschen Substantivphrasen für die deutsche Substantivphrase erstellt und auf die dänische übertragen wird. Das rezeptionsgrammatische Modell, das somit als Tertium Comparationis dient, ist ein lineares Modell, zumal der Textoberfläche (und damit der Topologie) eine entscheidende Rolle zugeteilt wird. Dies ist darauf zurückzuführen, daß die lineare, zweidimensionale Folge von Wörten und graphematischen Zeichen das einzige Konkrete ist, das dem Rezipienten bei der Rezeption zur Verfügung steht. Für die morpho-syntaktische Beschreibung der Substantivphrasen aus den aktuellen Fachtexten der Wirtschaft ist es jedoch unumgänglich, bei der Analyse auch auf die hierarchischen Strukturen der Substantivphrasen Rücksicht zu nehmen.

Dem rezeptionsgrammatischen Modell liegen vier Prinzipien zugrunde: das Phrasen-, das Klammer-, das Feld- und schließlich das Kettenprinzip.

Das Phrasenprinzip besagt, daß die hier zu beschreibende Wortgruppe als eine Substantivphrase gilt, weil sie unabhängig vom Kontext beschrieben wird (im Gegensatz zu den Satzgliedern) und ein Substantiv als Kern hat (Engel 1991).

Das Klammerprinzip besagt, daß für die Substantivphrase eine Klammer angenommen wird, die von dem Artikelwort und dem Kern der Substantivphrase gebildet wird. 
Das Feldprinzip besagt, daß die Substantivphrase eine Feldstruktur hat, die aus einem Artikelfeld, Vorfeld, Kernfeld, Schlußfeld, Zusatzfeld und darüber hinaus einem voran- und einem nachgestellten Extraktionsfeld besteht.

Das Kettenprinzip, das als das charakteristischste Merkmal des rezeptionsgrammatischen Modells anzusehen ist, besagt, daß die prototypischen Attribute im Schlußfeld (Genitivphrasen und Präpositionalphrasen) nur aus drei Feldern bestehen, und zwar aus dem Artikelfeld, dem Vorfeld und dem Kernfeld, die zusammen als Substantivrahmen beschrieben werden können. Kommen mehrere Attribute dieser Art vor, können auf diese Weise ganze Ketten von Substantivrahmen gebildet werden.

Als Analysedaten dienen ausschließlich maximale Substantivphrasen (SP), d.h. Substantivphrasen, "die nicht selber in Substantivphrasen eingebettet sind, sondern als Satzglieder (Kasusglieder) oder als regierte Nominalphrasen in präpositionalen Satzgliedern dienen" (Fabricius-Hansen / Solfjeld 1994, S. 43). Nicht alle SP werden jedoch bei der Analyse berücksichtigt, sondern nur diejenigen, die mit min. einer Erweiterung (außer einem evtl. Artikelwort) ausgebaut sind.

Der Evaluierung liegen folgende Thesen zugrunde:

1) Aus intralingualer Perspektive sind die SP der Schicht $C$ rezeptionsgrammatisch gesehen schwieriger zu verarbeiten als die SP der Schicht D.

2) Aus interlingualer Perspektive sind die SP der deutschsprachigen Texte aus der Schicht $\mathrm{C}$ rezeptionsgrammatisch gesehen schwieriger zu verarbeiten als die der dänischen Texte der Schicht C, und die SP der deutschsprachigen Texte aus der Schicht D schwieriger zu verarbeiten als die der dänischen Texte der Schicht D.

Als Parameter des Verarbeitungsgrads der SP gelten die SP im Verhältnis zu ausgewählten kontextuellen Größen (z.B. Anzahl von Perioden), der Ausnutzungsgrad der jeweiligen SP-Felder, die Breite (auch die Länge genannt), die Tiefe und das Material (sowohl syntaktisches Material wie z.B. GP und PP als auch Wortbildungsmaterial wie z.B. Nomen Actionis und Kompositum). Für rezeptionsgrammatisch gesehen besonders rezeptionserschwerend wird in diesem Zusammenhang das Vorkommen von Komposita, Nomina Actionis, nachgestellten GP (die systembedingt nur bei den deutschen SP vorkommen), Partizipialattributen, Erweiterungen vorangestellter Attribute und sogenannten Einschachtelungen (Heringer 1989) gehalten, weil diese Elemente im Vergleich zu diversen alternativen Ausdrucksmöglichkeiten schwieriger zu verarbeiten sind und somit besonders dazu beitragen, einen Text vergleichsweise schwerer zugänglich zu machen.

Anhand der durchgeführten Analysen lassen sich die aufgestellten Hypothesen bestätigen, und zwar sind die größten Unterschiede sowohl intra- als 
auch interlingual gesehen bei den besonders rezeptionserschwerenden Elementen zu verzeichnen. Was den Ausfüllungsgrad der einzelnen Felder betrifft, ist das Schlußfeld der deutschen SP aus interlingualer Perspektive öfter ausgefüllt als das der dänischen, und umgekehrt ist das Zusatzfeld der dänischen SP öfter ausgefüllt als das der deutschen SP. Gleiches gilt aus intralingualer Perspektive, und somit ist das Schlußfeld der deutschen SP der Schicht C öfter ausgefüllt als das der deutschen SP der Schicht D, und umgekehrt ist das Zusatzfeld der deutschen SP der Schicht D öfter ausgefüllt als das der deutschen SP der Schicht C. Dies könnte - was aus der Dissertation nicht deutlich hervorgeht - ein Indiz dafür sein, daß es im Dänischen bzw. bei der deutschen Schicht D eine stärkere Tendenz zur Verbalisierung gibt als im Deutschen bzw. bei der deutschen Schicht C, und korrelativ, daß es im Deutschen bzw. bei der deutschen Schicht $C$ eine stärkere Tendenz zur Deverbalisierung gibt als im Dänischen bzw. bei der deutschen Schicht D. Es wäre somit ein Indiz, das durch das häufigere Vorkommen von den deverbalisierten Ausdrücken Nomina Actionis und Partizipialattributen im deutschen Korpus generell bzw. bei den deutschen SP der Schicht C im Vergleich zum dänischen Korpus generell bzw. bei den deutschen SP der Schicht D unterstützt wird. Bezüglich der restlichen Parameter wie z.B. Breite und Tiefe lassen sich weder intra- noch interlingual wesentliche Unterschiede feststellen.

\section{Literatur}

Ahlgren, Bengt / Fabricius-Hansen, Cahtrine (1986): Å lese tysk sakprosa. En innføring i grammatisk leseteknikk. Oslo: Universitetsforlaget.

Ditlevsen, Marianne Grove (1996): Den udvidede substantivfrase i udvalgte tyske $\phi k o-$ nomiske fagtekster - en receptionsgrammatisk analyse i intra- og intersprogligt perspektiv. Unpubl. Diss., Wirtschaftsuniversität Aarhus.

Engel, Ulrich (1991): Deutsche Grammatik. Heidelberg: Julius Groos Verlag.

Fabricius-Hansen, Cathrine / Solfjeld, Kåre (1994): Deutsche und norwegische Sachprosa im Vergleich. Ein Arbeitsbericht (=Arbeitsberichte des Germanistischen Instituts der Universität Oslo $\mathrm{Nr}$. 6). Oslo.

Heringer, Hans-Jürgen (1989): Lesen lehren lernen. Eine rezeptive Grammatik des Deutschen. Tübingen (Studienausgabe).

Hoffmann, Lothar (1987): Kommunikationsmittel Fachsprache. Eine Einführung. Berlin: Akademie-Verlag Berlin. 